\title{
Transalpina
}

TRANSALPINA Études italiennes

$20 \mid 2017$

Edmondo De Amicis. Littérature et société

\section{Una galassia femminile : associazionismo laico nell'Italia del secondo dopoguerra}

\section{Patrizia Gabrielli}

\section{(2) OpenEdition}

12 Journals

\section{Edizione digitale}

URL: http://journals.openedition.org/transalpina/398

DOI: 10.4000/transalpina.398

ISSN: 2534-5184

\section{Editore}

Presses universitaires de Caen

\section{Edizione cartacea}

Data di pubblicazione: 1 septembre 2017

Paginazione: 243-258

ISBN: 978-2-84133-857-3

ISSN: $1278-334 \mathrm{X}$

Notizia bibliografica digitale

Patrizia Gabrielli, « Una galassia femminile : associazionismo laico nell'Italia del secondo dopoguerra », Transalpina [Online], 20 | 2017, online dal 19 décembre 2019, consultato il 07 novembre 2020. URL : http://journals.openedition.org/transalpina/398 ; DOI : https://doi.org/10.4000/ transalpina.398 


\section{UNA GALASSIA FEMMINILE : ASSOCIAZIONISMO LAICO NELL'ITALIA DEL SECONDO DOPOGUERRA}

Riassunto: L'intervento si incentra sull'immediato dopoguerra e intende offrire un quadro sull'impegno espresso sia dalle associazioni femminili sia da alcune singole protagoniste attive nel campo politico e culturale che, pur mantenendo il dialogo con le due principali associazioni femminili dell'Italia repubblicana, l'Udi e il Cif, manifestarono autonomia dai partiti di massa, rifiutarono la logica della polarizzazione affermatasi fin dai primi anni della guerra fredda ed ebbero un ruolo tutt'altro che secondario nel processo di ricostruzione del Paese. Sarà, dunque, rivolta attenzione alla "galassia» di associazioni e di soggetti (tra questi alcune intellettuali quali Alba de Céspedes), che operarono a favore della cittadinanza democratica e per l'affermazione di nuovi modelli di genere. Esse richiamarono l'attenzione dell'opinione pubblica anche su aspetti inerenti la morale ed il costume (alcuni accenni in tal senso saranno svolti a proposito di Anna Garofalo), e svolsero una vasta opera pedagogica e formativa finalizzata alla costruzione di una cittadinanza responsabile e consapevole.

Résumé: L'article est centré sur l'immédiat après-guerre et entend présenter un tableau de l'engagement tout à la fois des associations féminines et des femmes actives sur le plan individuel dans le domaine politique et culturel. Tout en maintenant le dialogue avec les deux principales associations féminines de l'Italie républicaine, l'Udi et le Cif, ces dernières firent preuve d'autonomie par rapport aux partis de masse, refusèrent la logique de la polarisation qui s'affirma dès les premières années de la Guerre froide, et jouèrent un rôle qui fut loin d'être secondaire dans le processus de reconstruction du pays. On s'intéressera donc ici à la "galaxie» des associations et des individus (dont certaines intellectuelles comme Alba de Céspedes) qui agirent en faveur de la citoyenneté démocratique et pour l'affirmation de nouveaux modèles de genre. Ces femmes attirèrent aussi l'attention de l'opinion publique sur des aspects relatifs à la morale et aux mours (avec en particulier des considérations à propos d'Anna Garofalo) et entreprirent une vaste action pédagogique et formatrice dans l'optique de la construction d'une citoyenneté responsable et consciente. 


\section{La rinascita}

Nel 1945 Anna Garofalo, scrittrice e giornalista, conduttrice della trasmissione radiofonica Parole di una donna, inserita nel milieu del liberalismo democratico, futura fondatrice del partito radicale, osserva che gli italiani, appena usciti dal dramma della guerra, manifestano « un immenso bisogno di parlare, dopo tanto silenzio, di comunicare con i nostri simili, di raccontar loro quello che avevamo sofferto, pensato, voluto e di sentirli parlare ${ }^{1}$.

Parlare, comunicare, agire, insieme alle grandi speranze verso il futuro, caratterizzano il fermento del dopoguerra, di cui si trova una delle più ampie espressioni nel 1946. È in questo anno che forze politiche e cittadini affrontano, non senza timori, le prime elezioni amministrative ed il referendum istituzionale ${ }^{2}$. Un fermento che Jolanda Vasely Torraca, tra le fondatrici del Consiglio nazionale delle donne italiane, restituisce in maniera esemplare nelle pagine del proprio diario: «fare qualcosa, uscire dalla pura contemplazione per non dover pensare, per non rivoltare in me tutti i problemi sociali e morali che questa guerra ha sollevato, $[\ldots] \mathrm{mi}$ devo agganciare in un'azione collettiva, devo utilizzare meglio la mia energia e la mia capacità di lavoro ${ }^{3}$. Se tra il 1944 e il 1945 nelle zone liberate si assiste alla mobilitazione dei partiti, alla eccezionale visibilità di quelli di massa con le nuove forme organizzative e di circolazione del « discorso politico ", contemporaneamente si va esprimendo un deciso protagonismo politico femminile sia all'interno alle singole forze sia in specifiche aggregazioni. Tra queste spiccano per numero di iscritte e presenza nel territorio l'Unione donne italiane (Udi), di matrice laica e legata al Partito comunista italiano (Pci), ed il Centro italiano femminile (Cif) nato in seno all'associazionismo cattolico e sostenitore della Democrazia cristiana (Dc). Sono queste organizzazioni a dominare la scena, come del resto il dibattito storiografico degli ultimi trent'anni, ma nel complesso palcoscenico del dopoguerra, esse non sono le uniche. Seppure meno visibili, e meno studiate in sede storica, sono presenti pure associazioni non assimilabili all'identità cattolica o comunista, indipendenti dai partiti politici e di vocazione laica, vicine alla tradizione liberale e azionista. Queste aggregazioni presentano un'identità sfaccettata, un'organizzazione poco strutturata fondata su una rete di relazioni amicali, di conoscenze talvolta di vecchia data, di rapporti informali al confine tra sfera pubblica e privata, sono nutrite e sostenute

1. A. Garofalo, L'italiana in Italia, Bari, Editori Laterza, 1956, p. 36.

2. 1946, $i$ comuni al voto, P. Dogliani, M. Ridolfi (dirr.), Imola, Mandragora, 2007.

3. Archivio Centrale dello Stato (d'ora in avanti ACS) Fondo Jolanda Torraca, b. 18. 
dalla condivisa consuetudine delle associate all'impegno civile ed alla vita associativa. Come ormai a qualche anno di distanza dal conflitto, sottolineava Anna Garofalo, queste associazioni e le singole personalità al loro interno avevano rappresentato una presenza più che significativa nello scenario dell'immediato dopoguerra: «lavoravano animate da pacifici obbiettivi come il lavoro, l'educazione, l'assistenza, la scuola, la posizione giuridica della donna e senza aver tendenze né estremiste né confessionali, si vedevano tutto il tempo ostacolate da interferenze politiche e scambiate per quel che non erano ${ }^{4}$. La rete di relazioni personali interna, che si dirama oltre i confini di ogni singola aggregazione, dà forma a un insieme dai contorni incerti e mobili, ad una "galassia» che in gran parte può essere collocata sia nella cornice della tradizione repubblicana, liberale e liberal socialista, definita «terza forza» in quanto collocata oltre la polarizzazione Dc-Pci, sia nel quadro della storia del femminismo.

L'impegno nel campo politico e sociale di queste donne, le tematiche principalmente affrontate, i valori, gli ideali e gli ostacoli sono le questioni cui rimanda Anna Garofalo nella citazione sopra riportata, che assumo quale filo conduttore per una possibile lettura e interpretazione di tali esperienze solo parzialmente analizzate dalla ricerca storica. Le pagine che seguono non hanno certo la pretesa di colmare questo vuoto, quanto di offrire qualche dato sulla ricca identità e sulle pratiche politiche dei movimenti delle donne del dopoguerra.

\section{Libertà, responsabilità, educazione civile}

Molte protagoniste dei movimenti femminili del secondo dopoguerra che confluiscono nell'associazionismo laico condividono posizioni antifasciste, sostanziate in molti casi da un vero e proprio tessuto di valori politici ed etici. La resistenza al nazifascismo, compiuta in forme diverse da quella armata, ha maturato un impegno in prima persona, un'assunzione di responsabilità verso la comunità di appartenenza. Elsa Dallolio, attiva nella Lega democratica di Gaetano Salvemini, segretaria generale della Croce Rossa Italiana, nel 1932 fondatrice della sezione italiana dell'International Social Service, definisce sinteticamente, ma con grande efficacia, questo slancio politico e ideale in una lettera all'amica Iris Origo. Scrittrice di origini inglesi, residente in Toscana, promotrice di un'intensa azione di solidarietà verso le popolazioni locali, che può essere assunta quale esempio di resistenza civile, la scrittrice è autrice, tra l'altro, di un bel libro

4. A. Garofalo, «La pace è di tutti», Il Ponte, VII, n. 1, gennaio 1951. 
che restituisce la statura morale e umana di Elsa Dallolio : «Cara Iris, sta' tranquilla per me. Mantengo il mio programma, se sarà possibile. Non si fa nulla di eroico e non credo certo di fare nulla di indispensabile, ma di stare come tutti sul piano della sorte di tutti, mi da il senso di stare al posto mio» ${ }^{5}$.

$\mathrm{Al}$ "proprio posto » vogliono stare le tante donne che popolano le associazioni femminili del dopoguerra, protagoniste della ricostruzione del Paese, le quali trasferiscono nella sfera pubblica attitudini e pratiche accumulate quotidianamente nella sfera privata, offrendo servizi a una popolazione sofferente. Esse sono al centro di un'opera diffusa e capillare che prevede, oltre alle attività solidali, la realizzazione di corsi, conferenze, dibattiti volti alla crescita civile delle italiane. Così come al «proprio posto » vogliono stare alcune intellettuali di orientamento liberal democratico e liberal socialista, donne fuori dagli schemi delle ideologie e dai partiti, sovente non inserite nell'Udi o nel Cif, seppure in stretto contatto con esse, che si impegnano in differenti «luoghi» per la difesa dei valori della democrazia e della libertà, intesi come non omologazione. Fautrici di un dialogo lontano da ogni integralismo, esse danno vita a riviste che polarizzano il confronto tra uomini e donne di grande statura intellettuale e politica. Costituiscono un esempio in tal senso «Fronte Unito» fondata e diretta da Fausta Cialente; "Città ", nata dall'impegno di Paola Masino e di altri intellettuali ${ }^{6}$; «Mercurio " giudicata « una delle migliori riviste politico letterarie [...] diretta da una donna, Alba de Céspedes ${ }^{7}$. Alcuni articoli della scrittrice, di origine cubana e di formazione cosmopolita ${ }^{8}$, favoriscono la comprensione dei compiti che le intellettuali dell'area liberale - declinata in un ampio ventaglio di orientamenti che vanno dal socialismo a posizioni moderate - attribuiscono alla politica.

Suffragio e partecipazione erano obiettivi condivisi da tutte, il cui raggiungimento imponeva di «fare i conti» con il fascismo, innescare un esame di coscienza collettivo per ricostruire e ricostruirsi : «Bisogna rifare

5. Cit. in I. Origo, Guerra in Val d'Orcia, Firenze, Vallecchi, 1968, cit., p. 70. Si veda della stessa, Ospitalità e fratellanza, in Italia e Gran Bretagna nella lotta di Liberazione (Atti del convegno di Bagni di Lucca, aprile 1975), Firenze, La Nuova Italia, 1977, p. 27-41.

6. Si veda L. Di Nicola, Intellettuali italiane del Novecento : una storia discontinua, Pisa, Pacini Editore, 2012. Su Fausta Cialente e la rivista si veda F. Rubini, «Fronte Unito 1943-1947. La Resistenza lontana», in Donne nelle minoranze, P. Gabrielli (dir.), Storia e problemi contemporanei, 68 , settembre 2015, p. 31-48.

7. A. Garofalo, L'italiana in Italia, p. 46. Sulla rivista si rimanda a L. Di Nicola, Mercurio: storia di una rivista 1944-1948, Milano, Il Saggiatore. Fondazione Arnoldo e Alberto Mondadori, 2012.

8. Si veda Alba de Céspedes, M. Zancan (dir.), Milano, Il Saggiatore. Fondazione Arnoldo e Alberto Mondadori, 2005. 
il popolo italiano » miglioralo occorre, insomma, «rifare anche noi stessi ${ }^{9}$, affermava Alba de Céspedes dimostrando di comprendere le tante difficoltà che si addensavano intorno al passaggio dal fascismo alla democrazia. Il rinnovamento politico, allora, passa attraverso il cambiamento dell'identità culturale del Paese, da qui la critica all'egoismo degli italiani che la scrittrice considera eredità del qualunquismo fascista, di un regime che, cancellando le libertà aveva inibito le scelte e la capacità di agire, l'assunzione di responsabilità verso la comunità di appartenenza, infranto i legami di solidarietà e favorito individualismo e familismo. E «l'eliminazione di ogni solidarietà di gruppo - è la conditio sine qua non del dominio totale ${ }^{10}$. Le pratiche di mobilitazione delle masse, miranti all'omologazione e al conformismo, avevano cancellato il senso civico già debole degli italiani e aperto un vuoto di valori civili: "Quando si demoliscono le paratie delle idee sociali e religiose con cui siamo state educate - annotava Jolanda Torraca - ci affacciamo ad un tratto ad un baratro vuoto che a molte fa girar la testa $»^{11}$. Compiti gravosi, ancor più difficili se si tiene conto delle avversioni generate da quel surplus di violenza proprio delle guerre civili e nel biennio 1945-46 tensioni e rancori sono tutt'altro che sopiti. Venuto meno autoritarismo e paternalismo fascista la società rischiava di sgretolarsi. Bisognava liberarsi anche dall'educazione fascista, da una cultura che gravava sulle coscienze delle generazioni maturate cresciute nel ventennio.

Responsabilità individuale $\mathrm{e}$ "rieducazione politica delle coscienze» ricorrono nelle parole di Alba de Céspedes come di Jolanda Torraca e si rinvengono nella progettualità politica della «terza forza».

A questa consapevolezza può essere ricondotta l'attenzione all'istruzione e alla formazione dei cittadini che segna la biografia di molte protagoniste di quella stagione.

Per Teresita Sandeski Scelba, Maria Comandini Calogero, Anna Garofalo, Anna Lorenzetto - solo per citarne alcune - il tema della responsabilità individuale è alla base della democrazia, secondo una visione che salda diritti e doveri, caposaldo del moderno paradigma della cittadinanza democratica. La richiesta del suffragio femminile si colloca allora lungo queste coordinate e resta saldamente ancorata al tema della libera espressione dell'individuo:

9. P. Gabrielli, "Italia combatte". La voce di Clorinda, in Alba De Céspedes, M. Zancan (dir.), Milano, Il Saggiatore. Fondazione Arnoldo e Alberto Mondadori, 2005, p. 266-306, la cit. è a p. 288.

10. H. Arendt, Introduzione, in Le origini del totalitarismo, H. Arendt, A. Guadagnin (dirr.), Milano, Bompiani, 1978, p. LXVII.

11. ACS, Fondo Jolanda Torraca, b. 18. 
È compito aperto specialmente a quei partiti di sinistra, soprattutto pensiamo al Pd'A, che pur garantendo un miglioramento effettivo delle condizioni economiche di queste donne, come membri della famiglia e come individui: si colorino nella fantasia di rossi meno fiammanti, assicurino l'autonomia della famiglia e propugnino la piena libera possibilità di espansione dell'individuo - che è con quello economico e inscindibile da quello - il presupposto base per avviare a un miglioramento il problema femminile italiano ${ }^{12}$.

Il problema era di natura culturale ed etica e il compito che le diverse forze politiche si trovavano ad affrontare non poteva esaurirsi nel suffragio ma in un impegno vasto:

Da secoli le donne sono considerate come schiave dei genitori, del marito, dei figli, della tradizione. Se di diritto o anche di fatto è ormai caduta tale schiavitù resta reale e intatta la mentalità della schiavitù : l'incapacità per la donna di essere qualcuno, di essere se stessa, di essere una persona che pensa e vuole e agisce perché ha il diritto, come il suo compagno, di intervenire nel modificare il proprio ambiente. [...]. Dunque rinnovamento strutturale e organizzativo di tipo socialistico per risolvere l'aspetto economico-sociale del problema che solo da tale avviamento può essere risolto; rinnovamento di carattere liberale e individualistico per risolvere quell'altro aspetto politico-sociale del problema che è legato non già all'economia, ma a una tradizione antiquata e del tutto illiberale del modo di vita ${ }^{13}$.

La libertà individuale intesa quale piena espressione della dignità, per liberali, azioniste, repubblicane si coniuga con l'assunzione della responsabilità pubblica e con la solidarietà, principi estranei e antagonisti al totalitarismo fascista, secondo un disegno che salda pieno riconoscimento della soggettività con la solidarietà sociale. Lo stesso stare al di là dei partiti di massa matura dall'esigenza di libertà e dall'anticonformismo: contrarie all'omologazione, che per molte aveva segnato la rottura con il fascismo e l'ingresso nella Resistenza, partendo dal valore dell'autonomia dell'individuo, le esponenti dell'azionismo e più in generale della «terza forza », proiettano il discorso sulla cittadinanza verso un orizzonte capace di tenere insieme aspetti di natura economica, sociale, culturale ed etica; esse si preoccupano di trasmettere un nuovo sentimento di appartenenza alla comunità nazionale. Lo studio delle tematiche inerenti la condizione femminile, inchieste, conferenze, convegni sono al centro

12. Ibid., M. Comandini Calogero, Considerazioni sul voto alle donne.

13. Ibid., M. Comandini Calogero, Il Partito d'Azione e il problema femminile 1945. 
del fitto calendario di attività. Tra le principali interpreti in tal senso Maria Comandini. Colta e istruita, proveniente da una famiglia di chiare idee repubblicane del cesenate, antifascista convinta, la giovane, che nel 1929 sposa Guido Calogero, è vissuta in Inghilterra e negli Stati Uniti. In questi paesi ella ha avuto modo di osservare e di conoscere altri sistemi e culture che le permettono di affinare le competenze sull'assistenza e sui servizi sociali, una tema quest'ultimo che è centrale nella sua biografia tanto da poter essere definita pioniera dell'assistenza in Italia. Per lei il tema è saldamente connesso con la costruzione della democrazia. Il fine dell'assistenza consiste, infatti, nell' «aiutare i meno capaci a far valere i loro diritti e sollecitarli a lavorare per un mondo migliore in cui ognuno sia impegnato a cooperare per la costruzione della comunità civile» ${ }^{14}$. Simili le finalità di un'altra liberale, Anna Lorenzetto, ricercatrice universitaria, che nel 1947 - anno in cui esce dall'Udi - fonda l'Unione nazionale per la lotta contro l'analfabetismo, riconosciuto dall'Unesco. Una scelta che la studiosa condivide con le liberali Josette Menasce Lupinacci e Teresita Sandesky Scelba e con la democristiana Maria De Unterrichter Jervolino. Anche per Anna Lorenzetto, sostenitrice dell'apprendimento di un "alfabeto maggiore», secondo un disegno che anticipa, per molti aspetti, la pedagogia e la prassi dell'educazione degli adulti ${ }^{15}$, la formazione dei cittadini è tra i compiti urgenti di quel dopoguerra. Non troppo lontana da questa impostazione è Margherita Zoebeli con il suo Centro educativo o "villaggio italo-svizzero" a Rimini, sostenitrice - come afferma Carlo De Maria, che alla pedagogista ha dedicato un'accurata biografia - di una «educazione attiva » e fiduciosa nella possibilità di costruire la democrazia a partire dall'educazione «intesa sia come lavoro con i bambini che come impegno degli adulti educatori a lavorare su se stessi» ${ }^{16}$.

Nel clima infuocato del dopoguerra, in un Paese che contava molte ferite aperte, dove si annidava un profondo disagio esistenziale, singole personalità e associazioni si esprimono al di là del collateralismo; prendono parte attiva, attraverso la erogazione di incentivi alla partecipazione, al difficile processo di integrazione delle italiane alla cittadinanza democratica. Da queste posizioni maturano iniziative volte all'educazione della cittadina, che trovano la loro più visibile espressione in occasione delle

14. P. Benvenuti, D.A. Gristina, La donna e il servizio sociale, Milano, FrancoAngeli, 1998, p. 94 .

15. A. Lorenzetto, «La lotto contro l'analfabetismo e il problema dell'educazione degli adulti», Il Ponte, 6/5, 1950, p. 455-470.

16. C. De Maria, Il lavoro di comunità e ricostruzione civile in Italia. Margherita Zoebeli e il centro educativo italo-svizzero di Rimini, Roma, Viella, 2015, p. 16. 
campagne elettorali del 1946, quella per le amministrative, che si svolgono in marzo-aprile nelle regioni del Centro-Nord, e quella per il referendum istituzionale e l'elezione dell'Assemblea Costituente il 2 giugno.

\section{Il Comitato Pro Voto}

Almeno un altro tratto, insieme alla consapevolezza dei tanti problemi che si addensano intorno al passaggio dal totalitarismo fascista alla democrazia, compresa la necessità di ridare spazio e agibilità politica alle donne, distingue molte animatrici dell'associazionismo laico : il legame con il femminismo di primo Novecento. Un rapporto che emerge con forza dalla scelta di porre al centro delle proprie elaborazioni e rappresentazioni l'identità di genere, perno intorno al quale s'incardina il progetto di un'ampia alleanza tra donne di diversa appartenenza politica, di classe, religiosa. Esse sono per molti versi le eredi del femminismo. Un lascito e una preziosa genealogia femminile che risultano sostanzialmente estranee alle giovani generazioni, come acutamente osservava, nel 1946, Anna Franchi, socialista e scrittrice, nel suo Cose d'ieri dette alle donne di oggi ispirato alla volontà di non disperdere la memoria storica del femminismo italiano e alla consapevolezza che la tradizione è a fondamento della legittimazione politica ${ }^{17}$.

Tra le principali associazioni che fanno tesoro di memoria, modelli e pratiche del femminismo spiccano l'Alleanza femminile italiana (Afi) e il Consiglio nazionale della donna italiana (Cndi).

L'Afi, fondata nel 1909, erede della Pro suffragio, sostenitrice del voto, del divorzio, dell'abolizione dell'insegnamento religioso nelle scuole ${ }^{18}$, vide tra le sue principali animatrici Teresita Sandeski Scelba, nata a Torino nel 1885, da madre italiana e padre di origini polacche, presto si trasferisce a Roma dove si laurea in medicina nel 1909 e si impegna in varie iniziative in favore dell'igiene sociale. È lungo questo filone di attività - comune ad altre figure del femminismo - che Teresita, ancora giovanissima, approda all'Associazione per la donna e all'Alleanza femminile ed al Cndi di cui assume la vicepresidenza. Intanto si va meglio definendo la sua dimensione professionale. Nel 1915 Sandeski dirige l'Ufficio d'Igiene della capitale, incarico che manterrà fino al 1955, anno del suo pensionamento. Nel 1944, in una Roma appena liberata e tragicamente segnata dalla violenza della

17. A. Franchi, Cose d'ieri dette alle donne d'oggi, Milano, Hoepli, 1946. Ho avuto modo di soffermarmi su questi aspetti nel mio La pace e la mimosa. L'Unione donne italiane e la costruzione politica della memoria (1944-1955), Roma, Donzelli, 2005.

18. T. Sandeschi Scelba, Teresita Sandeschi Scelba e i suoi tempi, Roma, Consiglio nazionale delle donne italiane, 1975 . 
guerra, ella va alla ricerca delle amiche femministe di vecchia data, tutte ormai anziane: «assieme a esse convoca delle riunioni in case private, entra in contatto con le rappresentanti dei partiti antifascisti rientrate dall'esilio o uscite dalle carceri, raduna un piccolo gruppo e indice un incontro con l'obiettivo di ridare vita all'associazione ${ }^{19} \mathrm{e}$ "per ridare vita e posto adeguato al problema femminista $»^{20}$. Nella stessa città e negli stessi mesi, si va impegnando in tal senso una conoscente di vecchia data di Teresita, Jolanda Vesely Torraca. Nata nel 1902 nella provincia di Piacenza, anche lei di origini straniere - entrambi i genitori cecoslovacchi - trascorre l'infanzia in diverse città italiane; successivamente è a Praga dove si impiega presso l'Ambasciata Cecoslovacca. Tornata in Italia, anche in virtù dei legami del marito Vincenzo Torraca, giornalista antifascista, stringe relazioni con personaggi vicini a Giovanni Amendola, Gaetano Salvemini, Umberto Zanotti Bianco, Giuliana Benzoni ed Elsa Dallolio - solo per citare una parte della sua rete di conoscenze e frequentazioni - e s'impiega presso la Biblioteca dell'Associazione per gli interessi del Mezzogiorno. A ridosso della liberazione di Roma Jolanda è nominata dal Commissaria prefettizia all'Opera Assistenza Maternità e Infanzia, ente morale fondato nel 1917 dall'Associazione della donna, successivamente inglobato dalle politiche assistenziali del regime fascista. Ella confida in più occasioni sulle competenze mediche di Teresita che le fu d'aiuto di fronte ai gravi problemi sanitari che assillavano i ricoverati del dispensario. I rapporti tra le due riprendono rafforzati dal comune impegno per la riorganizzazione del Consiglio nazionale delle donne italiane, nato nel 1903, poi sciolto, dopo una breve fase di allineamento con il regime fascista guidata dalla marchesa Daisy de Robillant. Un processo di ricostruzione per il quale Teresita e Jolanda lavorano sodo insieme con le repubblicane Giuseppina Capurro Picchi ed Elisa Malintoppi, e con Nina Ruffini, medaglia alla Resistenza e riconoscimento della Legione d'onore francese. Nominata alla Segreteria Generale del Consiglio, Jolanda Torraca vi dedicherà impegno ed energie: «Ho amato molto il Consiglio e gli ho dedicato più di trent'anni della mia esistenza proprio per quella sua idea un po' assurda e idealistica di unire le donne di tutto il mondo, senza distinzione di razze e di religioni, in uno sforzo di vicendevole comprensione $»^{21}$.

19. M.A. Serci, «L'Alleanza femminile italiana 1944-1950. Per una legge contro lo schiavismo sessuale delle donne", in Donne nelle minoranze, p. 65-89.

20. T. Sandeschi Scelba, Teresita Sandeschi Scelba e i suoi tempi, p. 13.

21. J. Torraca, La mia storia. Seguito da Diario di una sedicenne d'altri tempi, Roma, Epsylon, 2013, p. 25. 
La composizione politica dell'Alleanza e del Cndi è poliedrica, vi confluiscono repubblicane, azioniste, liberali. Alto il livello d'istruzione delle aderenti, molte delle quali vantano anche periodi di istruzione o di permanenza all'estero o comunque un certo cosmopolitismo. La maggioranza delle socie è laureata, qualcuna possiede competenze giuridiche. Fra gli scopi condivisi la promozione di riforme morali, giuridiche ed economiche, finalizzate alla parità uomo-donna; significativi gli interventi sulla prostituzione, questione centrale nella storia del femminismo nazionale e internazionale e tra i problemi più sentiti in quel tragico dopoguerra ${ }^{22}$. Implementata negli anni anche l'attività di studio e di ricerca sulle questioni inerenti la vita pubblica, professionale ed economica, delle donne. Ma nell'immediato dopoguerra è il suffragio a catalizzare l'attenzione delle cattoliche, delle social-comuniste come delle laiche.

La guerra è ancora in corso, quando, nelle zone liberate, tra l'autunno del 1944 e il gennaio dell'anno successivo, questa rivendicazione torna ad animare con forza il dibattito e le attività dell'associazionismo femminile di diverse tendenze, che si impegna senza risparmio di energie. Se la volontà dei partiti - come in più occasioni e da più parti si è osservato - rappresenta un fattore significativo per il conseguimento di questo diritto, non è però da sottovalutare l'intervento delle donne: «l'azione per ottenere il diritto di voto - ha ricordato Maria Federici, prima Presidente del Cif - si sviluppò nel 1945 per opera di associazioni femminili e di singole persone e fu la prima scuola di educazione politica, attraverso la quale la donna maturò rapidamente la sua coscienza civica ${ }^{23}$. La consapevolezza che la partita sul voto fosse tutt'altro che scontata condusse donne di differenti orientamenti, persino opposti, alla promozione di un articolato calendario di iniziative, molte delle quali unitarie. In questa cornice, una delle più importanti risale al 25 ottobre 1944, quando le rappresentanti dei Comitati femminili del Pci, Psiup, Pd'A, Pli, Sinistra cristiana e Democrazia del lavoro, la Federazione italiana laureate (Fildis), l'Alleanza femminile, danno vita al Comitato Pro voto, capace di rievocare, almeno alle più anziane, il biennio 1904-1906, pagina d'oro per la storia del femminismo italiano, quando viene istituito un Comitato unitario per il suffragio a sostegno della petizione, che vide

22. Sull'impegno del femminismo italiano in questo ambito si veda almeno A. Buttafuoco, Le mariuccine. Storia di un'istituzione laica: l'asilo Mariuccia, Milano, FrancoAngeli, 1985; per un quadro sul dopoguerra e l'Italia repubblicana si rimanda a S. Bellassai, La legge del desiderio. Il progetto Merlin e l'Italia degli anni Cinquanta, Roma, Carocci, 2006.

23. M. Federici, L'evoluzione socio-giuridica della donna alla Costituente, in Studi per il ventesimo anniversario dell'Assemblea costituente, vol. II, Firenze, Vallecchi, 1969, p. 199-225, la citazione è a p. 215. 
quali prime firmatarie Anna Maria Mozzoni e Maria Montessori, presentata nel marzo del 1906 al Parlamento.

Nel 1944 il valore della scelta unitaria è sottolineato da più parti:

oggi hanno parlato alla radio le rappresentanti del Comitato femminile Pro voto, e hanno lanciato un appassionato messaggio alle donne delle zone ancora occupate: «Noi donne dell'Italia libera stiamo conducendo una battaglia che è per la libertà di tutte, per il progresso di tutte: la battaglia per il diritto di voto. I compiti per la ricostruzione sociale, politica, amministrativa del paese sono stati finora ripartiti fra la popolazione maschile, ma noi che abbiamo resistito e combattuto per affrettare il ritorno della libertà abbiamo il diritto e il dovere di non essere soltanto spettatrici di questo rinnovamento. Pensiamo che la nostra opera sia necessaria ora come fu necessaria allora. Il giorno in cui saremo tutte riunite dovrà segnare una ripresa dell'attività comune, sancita da una legge che ci consideri pienamente cittadine nel nostro paese $»^{24}$.

I sacrifici sopportati e le attitudini mostrate durante il tragico conflitto bellico, l'esempio di altri paesi, sono tematiche prevalenti nel dibattito, vi si appella pure il Comitato Pro voto nel promemoria ai partiti antifascisti spronati a sostenere presso il governo il suffragio femminile.

Nei pochi mesi che precedono l'emanazione del decreto sul voto, si assiste, dunque, ad una decisa azione da parte del Pro suffragio che le forze politiche, in parte già orientate, non possono ignorare. Una sintesi in tal senso è ben articolata da Maria Comandini Calogero, responsabile del movimento femminile del Partito d'Azione:

Non si può negare che questo diritto sia stato riconosciuto più per opera dei partiti, che da esso contano di trarre grandi vantaggi elettorali, che non da una vera e propria agitazione popolare che abbia obbligato il governo a questa concessione. Ma è anche vero che di fronte all'imponente opera compiuta dalle associazioni femminili di resistenza, e di fronte alle notevoli forze femminili organizzate nelle associazioni di massa - o nei movimenti di partito - anche i partiti che avrebbero potuto opporsi - e con plausibili argomenti, dopo il ventennio di campagna demografica - hanno sentito che, in questa nuova situazione, non potevano farlo. L'aria era cambiata e radicalmente ${ }^{25}$.

24. A. Garofalo, L'italiana in Italia, p. 25.

25. AMCC, M. Comandini Calogero, Considerazioni sul voto alle donne, s. d. 


\section{Il decreto}

La mobilitazione è in pieno svolgimento, quando il secondo governo Bonomi emana il $1^{\circ}$ febbraio 1945 il decreto luogotenenziale che estende il diritto al suffragio alle italiane. Stando alla storiografia la scelta del decreto nasconde l'intento conservatore sostenuto dal timore che dalla mobilitazione femminile, che comprendeva anche una petizione, potessero emergere altre rivendicazioni. Il timore era ben fondato, lo testimoniano gli articoli e gli interventi delle dirigenti di diversi orientamenti :

la battaglia per l'estensione del voto alle donne, vinta sul piano giuridico, non è certo vinta sul piano sostanziale. Si tratta, infatti, di una battaglia da portare sul piano del costume, della arcaica mentalità ancora diffusa e prevalente $[\ldots]$ che ha impedito e impedisce tuttora alle donne italiane di farsi creature autonome, con una volontà propria, una indipendenza di pensiero, una reale cittadinanza nella società ${ }^{26}$.

Lo sviluppo di un dibattito sul voto, in sintesi, avrebbe immesso sul palcoscenico della politica altri diritti e altri modelli di cittadinanza, dato confermato, seppure con delle differenze, con punte moderate o radicali, dai programmi delle associazioni femminili, stando ai quali l'accesso delle donne alla sfera politica non è una semplice inclusione, ma è portatore di nuove domande che modificano lo stesso paradigma della cittadinanza e ne ampliano il campo semantico.

Il diffuso antisuffragismo accompagnato da un generale clima di restaurazione dei tradizionali ruoli di genere, riduce il suffragio a « concessione» $\mathrm{o}$ a "premio" svuotandolo dei contenuti di cui lo ha invece riempito il fronte suffragista ${ }^{27}$. Opposizione o indifferenza con le quali devono misurarsi pure le donne di «terza forza» che trovano un clima poco benevolo ${ }^{28}$. Limite individuato con acume da Anna Garofalo che lo considera al pari di una vera e propria occasione mancata: «quei partiti laici, di tradizione risorgimentale, che avrebbero potuto, dalla Liberazione in poi, attrarre grandi

26. AMCC, M. Comandini Calogero, minuta di articolo per «La Voce Libera di Trieste»; si veda anche M. Comandini Calogero, «Il Partito d'Azione e il problema femminile», $\mathrm{La}$ Rinascita, 2 settembre 1945.

27. A. Rossi-Doria, Diventare cittadine. Il voto delle donne in Italia, Firenze, Giunti, 1996. Medesima la tesi di P. Gaiotti De Biase, si veda Il voto alle donne, in Democrazia cristiana e costituente (Atti del convegno di studio tenuto a Milano il 26-28 gennaio 1979), vol. I, Le origini del progetto democratico cristiano, G. Rossini (dir.), Roma, Cinque Lune, p. 415-456.

28. Si veda N. Crain Merz, L'illusione della parità. Donne e questione femminile in Giustizia e Libertà e nel Partito d'azione, Milano, FrancoAngeli, 2013. 
masse di donne, specie del ceto medio e intellettuale e non hanno saputo farlo ${ }^{29}$. I partiti che attribuivano al diritto di voto un valore anche sul piano della libertà individuale si mostrarono «i più indifferenti o diffidenti verso il voto alle donne» ${ }^{30}$.

In occasione delle celebrazioni del decennale, nel 1955 a Roma, Teresita Sandesky Scelba tornava con forza sull'evento, negando la tesi della «concessione» ella poneva in risalto il protagonismo femminile:

È stato detto che le donne non hanno voluto il voto, che il voto è stato regalato. Desidero anzitutto togliere questa idea dalla testa di coloro che, non avendo fatto parte della vita femminile italiana, possono crederlo. Il voto è stato richiesto razionalmente e internazionalmente, tanto è vero che se il Cndi è stata la prima associazione internazionale e poi nazionale italiana (fondata nel 1888) tra donne, è venuta molto presto (1904) l'Alleanza per il Suffragio femminile, associazione internazionale alla quale si è immediatamente affiliata l'associazione per il Suffragio femminile italiana. È stata una lotta di molti anni, è stata una lotta ripresa immediatamente dopo la Liberazione. Se quindi il voto ci è stato dato, è stato preparato da queste donne tanto in Italia quanto all'estero ${ }^{31}$.

A dieci anni di distanza, le testimoni si esprimono senza mezzi termini su quel processo di stratificazione della memoria che aveva ridotto il suffragio a concessione consapevoli che ciò aveva indebolito sul piano simbolico l'esercizio di quel diritto ed il dibattito intorno ad esso. Nell'oblio era caduta anche l'azione unitaria del Comitato Pro voto e le biografie di tante sue protagoniste. Sempre nel 1955, interpretando la rottura dell'unità che aveva fatto seguito alla guerra fredda come una vera e propria occasione mancata, Teresita Sandesky richiamava la capacità di alleanza espressa nell'immediato dopoguerra:

Ed è infinitamente doloroso, dopo aver visto, anzi meglio dopo aver vissuto, nel periodo tra il 1944 e il 1945 l'intensa, concorde attività delle donne di diversissima provenienza, per ottenere unite il riconoscimento del loro diritto al voto, è doloroso che quella unione sia stata spezzata. Questo mio rincrescimento non ha solo un valore, diciamo così, sentimentale; io rimpiango, l'atmosfera cordiale, solidale della nostra comune azione, ma rimpiango ancor di più i risultati che senza dubbio avremmo ottenuto in

29. A. Garofalo, L'italiana in Italia, p. 83.

30. A. Rossi-Doria, Diventare cittadine, p. 42.

31. G. Saija, «Il voto non ci è stato regalato!», Noi donne, 23 aprile 1955. 
tutti i campi che interessano le donne, se le donne fossero rimaste unite nel difendere i loro diritti ${ }^{32}$.

Non distanti da quelle di Sandesky le osservazioni e i ricordi di Josette Menasce Lupinacci, responsabile del movimento femminile liberale e socia del Cndi :

Per me quello che conta è lo spirito con il quale allora abbiamo lottato le une a fianco delle altre, liberali, socialiste, comuniste, democristiane, repubblicane, donne diversissime per origine, educazione, tendenze, ma unite allora tra loro da una comune convinzione: che era necessario che le donne italiane uscissero finalmente da uno stato di inferiorità politica e sociale e partecipassero insieme agli uomini alla ricostruzione dell'Italia. Del resto nessuno poteva in quei mesi negare che le donne partecipavano già, attraverso il loro immenso, eroico contributo alla Resistenza, a cancellare vent'anni di fascismo e a lavorare al rinnovamento del paese. Questo è in me il ricordo più vivo la nostra solidarietà, l'affiatamento per cui non ci consideravamo più soltanto tra noi alleate ma amiche ${ }^{33}$.

Lo scontro ideologico e di contrapposizioni della guerra fredda esaspera il clima politico introducendo altri schemi: prevale l'antinomia amico-nemico mentre la "guerra civile dell'anima " ${ }^{34}$ tocca il femminismo, spazzando via ogni possibilità di collaborazione tra Udi, Cif e associazionismo laico. Tra il 1947 ed il 1948 l'Udi subisce una vera emorragia di adesioni. Un processo al quale ha fatto riferimento anche Jolanda Torraca raccontando la sua personale esperienza:

ben presto feci parte del Consiglio Nazionale [dell'Udi] nel quale figuravano repubblicane, socialdemocratiche e perfino alcune liberali. Ma qualche anno dopo ne uscimmo tutte insieme quando l'Udi, sotto la presidenza di Maria Maddalena Rossi, succeduta a Rita Montagnana, allora moglie di Togliatti, cadde completamente in mano al Partito comunista ${ }^{35}$.

Vale però la pena aprire una riflessione anche sulle difficoltà e sul nuovo assetto politico e sociale del dopoguerra. La partecipazione delle donne, compresa la mobilitazione Pro voto, frutto della nuova consapevolezza maturata dalla guerra e dalla partecipazione alla Resistenza, sostenuta in

32. Ibid.

33. G. Saija, «Un legame che non si è spezzato », Noi donne, 9 gennaio 1955.

34. R. Bodei, Il noi diviso. Ethos e idee dell'Italia repubblicana, Torino, Einaudi, 1988.

35. J. Torraca, La mia storia, p. 142. 
molti casi da un sincero sentimento di appartenenza di genere, si intrecciò con la presenza di due nuove variabili. Lo scenario del dopoguerra è dominato da almeno due novità, i partiti di massa e il suffragio femminile, entrambi segno dei processi di modernizzazione visibili nelle società europee a partire dalla grande guerra, che infrangono i vecchi meccanismi della politica e formulano nuovi, altri standard di aggregazione diametralmente opposti ai precedenti e decretano l'impossibilità di un ritorno indietro, ovvero il ripristino di organizzazioni confacenti a modelli politici elitari. Questi processi travolgono pure la possibilità di ripristinare le forme sperimentate dal primo femminismo, unioni fondate sull'appartenenza di genere. I partiti sono considerati strumenti d'intervento politico, mezzi atti a stimolare, a convogliare la partecipazione e fare fronte alla frammentazione della società. Soprattutto per le antifasciste, che formavano il ceto politico femminile dell'Italia repubblicana, essi apparivano alla stregua di una riconquista, dato che il fascismo, dopo aver decimato i militanti con la repressione e la violenza, li aveva sciolti.

Nei primi anni del dopoguerra l'associazionismo femminile, pur mantenendo intenti unitari su molti temi, è segnato da forti appartenenze politiche, ma il quadro di riferimento era fortemente mutato rispetto ai primi anni del secolo: ormai le donne votavano e non potevano non schierarsi, sostenere i propri partiti con i rispettivi candidati e candidate.

A questo nuovo quadro, oltre che agli ostacoli, alle resistenze, ai travisamenti e ai pregiudizi verso le femministe, cui fa riferimento Anna Garofalo, è da attribuire la debole presa dell'associazionismo liberale. Nonostante il debole radicamento, l'Afi ed al Cndi, ebbero una funzione di stimolo sulle più ampie formazioni di matrice laica e cattolica, furono capaci di tenere vivi rapporti e scambi internazionali.

Anche solo per queste ragioni, lo studio di queste espressioni minoritarie è significativo. L'analisi dei programmi e degli interventi di queste forze potrebbe portare alla luce l'ampiezza del dibattito sulla cittadinanza avviato dalle donne, le eventuali sollecitazioni sulle elette all'Assemblea Costituente impegnate nell'elaborazione di un testo costituzionale garante dell'uguaglianza di genere. Ma vi è almeno un'altra questione che meriterebbe di essere affrontata, individuabile fino dal primo approccio allo studio di questa "galassia femminile» : mi riferisco sia all'internazionalizzazione ed alla fitta rete di relazioni con altri paesi sia alla vena cosmopolita che le anima. Un dato che sollecita la riflessione sull'ingresso in Italia di nuove elaborazioni e pratiche politiche. Il contatto con le femministe di altre nazionalità e culture, con realtà più avanzate sul piano del riconoscimento della parità di genere contribuì ad estendere gli orizzonti politici, fu un'occasione preziosa di maturazione, di comparazione con 
esperienze più progredite. Si tratta di temi e questioni che implicano una specifica attenzione alla dimensione transnazionale dei movimenti delle donne e conducono al cuore della Women's Global History, un filone di studi che costituisce una delle punte più innovative nell'attuale dibattito storiografico sul Gender ${ }^{36}$.

Patrizia GABRIELLI

Università degli Studi di Siena - sede di Arezzo

36. Per un quadro in tal senso Women's History in Global Perspective, 3 voll., Urbana, University of Illinois Press, 2004-2005; Connected Worlds. History in Transnational Perspective, A. Curthoys, M. Lake (dirr.), Canberra, Australian National University Press, 2005; Women's Activism. Global Perspective from the 1890 s to the Present, F. de Haan, M. Allen, J. Purvis, K. Daskalova (dirr.), London - New York, Routledge, 2013; C.E. DuBois, K. Oliviero, "Circling the Globe. International Feminism Reconsidered, 1920 to 1975", Women's Studies International Forum, 32, n. 1, 2009; Per quanto concerne la dimensione internazionale del femminismo italiano costituisce un valido esempio E. Guerra, Il dilemma della pace. Pacifiste e femministe sulla scena internazionale, 1914-1939, Roma, Viella, 2014; si vedano anche M.C. Giuntella, Il protagonismo femminile internazionale, in Donna e modernità, E. Cavalcanti (dir.), Roma, Edizioni Dehoniane, 1993; Cittadine d'Europa. Integrazione europea e associazioni femminili italiane, B. Pisa (dir.), Milano, FrancoAngeli, 2003; A. Scarantino, Donne per la pace. Maria Bajocco Remiddi e l'Associazione internazionale madri unite per la pace nell'Italia della guerra fredda, Milano, FrancoAngeli, 2006. 